\title{
Association between High Cumulative Numbers of Elevated Heart Rate and Mortality in Neurological ICU patients: Retrospective Analysis of elCU Collaborative Research Database
}

\section{Dawei Zhou}

Beijing Tiantan Hospital https://orcid.org/0000-0002-7818-0107

Zhimin Li

Beijing Tiantan Hospital

Shaolan Zhang

Beijing Tiantan Hospital

Jianxin Zhou

Beijing Tiantan Hospital

Guangzhi Shi ( 15866601260@163.com)

Research article

Keywords: eICU-CRD, NICU, heart rate, APACHE IV score, mortality

Posted Date: April 22nd, 2019

DOl: https://doi.org/10.21203/rs.2.9183/v1

License: (c) (i) This work is licensed under a Creative Commons Attribution 4.0 International License.

Read Full License 


\section{Abstract}

Background Heart rate is routinely measured in Neurological intensive care unit(NICU), but its prognostic value remains debated. We sought to evaluate the association of high cumulative numbers of elevated Heart Rate (HcneHR) with mortality in NICU patients. Methods We used a large observational elCU Collaborative Research Database (eICU-CRD), where continous heart rate monitoring every 5 minute was available. We collected periodic heart rate, disease severity (APACHE IV score), NICU and hospital mortality and other information in 8347 patient admissions from the eICU-CRD. The cumulative numbers of Heart Rate (cneHR) were defined as $>100$ beats/min in first admittion 24 hours, and if cneHR $\geq 10$,then was defined as higt cneHR(HcneHR). The primary outcome was NICU mortality. The other outcomes were hospital mortality, length of NICU stay and APACHE IV score. Multivariable logistic regression was used to assess for association for HcneHR and other covariance with NICU and hospiltal discharge status. Results The mean age of patients were 63 years, and the most frequent disease categories of NICU in elCU-CRD were postoperation (25\%), stroke(19\%), traumatic brain injury (14\%). The mean APACHE IV score was 50 . Overall NICU mortality of the cohort at discharge was $4 \%$, and hospital mortality was $8 \%$. The NICU mortality of HcneHR patients was 7\%. Adjusted logistic regression for HcneHR showed a significantly increased risk of NICU death with odds ratio 1.61(confidence interval, 1.26-2.06; $P<0.001$ ). Conclusions In adult neurocritically ill patients, we found a significant association for HcneHR with elevated mortality and several others important patient-centered outcomes.

\section{Background}

The burden of critical illness is higher than generally appreciated and will increase as the population ages, preventive and therapeutic interventions that are generalisable across ICUs are needed[1]. Heart rate is routinely measured in ICU but its prognostic value remains debated. It is well known that critically ill patients are prone to develop tachycardia due to various conditions such as anxiety, pain, fever, infection, hypovolemia, exaggerated sympathetic activation, heart failure, hypoxia, anemia, drug effects, primary arrhythmia and so on[2-4]. It has been shown that there is an association for high heart rate(HR) and poor outcomes in critically ill patients[5], and HR is an important part of most ICU prognosis scores[6].

The critical care unit is a dataintensive environment[7]. However, as yet, heart rate from bedside monitor in critical care is evaluated by clinicians almost in much the same way as before, little information is available on the critical duration of tachycardia, especially in neurological intensive care unit(NICU), which focuses on the optimal management of acutely ill patients with life threatening neurologic and neurosurgical disease or with life-threatening neurologic manifestations of systemic disease[8]. We may get more information on the continuous heart rate monitoring.

Therefore, in this study, we hypothesized that an elevated HR of $>100$ beats/min for a prolonged period, where we showed it as high cumulative numbers of elevated $\mathrm{HR}(\mathrm{HcneHR})$ during the first day of NICU stay, may be associated with decreased survival in neurocritically ill patients. This may help clinicians to better identify high risk patients admitted in NICU. 


\section{Methods}

\section{Setting}

This study used data stored in the high-resolution database, the eICU-CRD (eicu-crd.mit.edu), which comprises 200,859 patient unit encounters for 139,367 unique patients admitted between 2014 and 2015. The database contains parameters that were available in the ICU clinical information system, including vital signs, laboratory measurements, medications, APACHE components, care plan information, admission diagnosis, patient history, time-stamped diagnoses and similarly chosen treatments. The elaborate description of elCU-CRD is available elsewhere[9].

The eICU-CRD was exempt from institutional review board(IRB) approval due to the retrospective design, lack of direct patient intervention, and the security schema, for which the re-identification risk was certified as meeting safe harbor standards by Privacert (Cambridge, MA) (Health Insurance Portability and Accountability Act Certification no. 1031219-2). Due to the HIPAA compliant de-identification in this database, our institutional IRB requirement was waived. After completing a National Institutes of Health(NIH) web-based training course(Protecting Human Research Participants), the author(dw zhou, certification number: 28795067) was approved to access to the database for research aims.

\section{Study Population}

All patients in the elCU-CRD were eligible for inclusion in the present investigation. As for those who admitted in NICU for more than once, we considered them as different patients. We selected all adults patients admitted in NICU with length of stay $>24$ hours, and excluded those who used heart rate controlling drug during the first day in NICU. Heart rate controlling drug contained $\beta$-blocker and calcium antagonist. We collected all periodically recorded heart rates for the first 24hours of NICU admission, and patients without periodical heart rate data were excluded. Patients without APACHE IV score, pulse oxygen saturation(Sp02), admission temperature, admission blood pressure, gender information were excluded.

\section{Clinical Variables and Outcomes}

Data on the following information during the first 24 hours of admission were extracted: age, gender, ethnicity (caucasian, african american, hispanic, asian, ative american, other/unknown), admission disease categories (postoperation, ischemic stroke, traumatic brain injury, intracranial hemorrhage, seizures, sepsis, subarachnoid hemorrhage, coma, others), admission vital sign(temperature, respiratory rate, heart rate, mean blood pressure, SpO2 ), heart rate related indexes for continuously periodical monitoring(cneHR, HcneHR, maximum heart rate, minimum heart rate, average heart rate), acute physiology score, APACHE IV score, the first day hemoglobin, comorbidities, including atrial fibrillation, cardiovascular diseases, tracheal intubation, mechanical ventilation, vasopressors, length of NICU stay, the NICU and hospital discharge status(alive or expired). All patients admitted from the operating room or 
recovery room had a surgical diagnosis, even though some surgical patients had a medical reason for admission to the NICU.

Periodical data refers to data which is consistently interfaced from bedside vital signs monitors into eCareManager. Continuously measured heart rate was originally collected at 1-minute intervals, with 5minute medians archived in elCU-CRD. That is to say, every patient in the first day of NICU had 288 heart rate values. The cneHR was defined as cumulative numbers of heart rate $>100$ beats $/ \mathrm{min}$ in first admission 24 hours archived in elCU-CRD "vitalPeriodic" table, and if cneHR (10,then was defined high cneHR(HcneHR), and cneHR<10 was defined as Low cneHR(LcneHR).

The primary endpoint of our study was NICU mortality, defined as death observed during NICU stay. Other study endpoints included hospital mortality, NICU length of stay and APACHE IV score. NICU length of stay was defined as the difference between date of NICU admission and discharge.

\section{Statistical Analysis}

Continuous variables are shown as mean (standard deviation) or median (interquartile range), and were analyzed with Wilcoxon rank-sum tests. For dataset with a large sample size, the Anderson-Darling test can be very sensitive to a small deviation from the normal distribution. So we switched off the normality testing and still used mean and standard deviation to describe the data[10]. Categorical variables were reported as numbers and percentages, and were analyzed with chi-square test. As for missing data onto the first day hemoglobin, we use the mean hemoglobin of the NICU alive patients to imputed the missing data onto the NICU alive patients, so was for the NICU expired patients. Some patients did not get the heart rate monitoring immediately after admission to $\mathrm{NICU}$, the missing heart rate data were replaced using next observation carried backward(NOCB) method.

Receiver operating characteristic (ROC) analysis was used to evaluate the predictive ability of cneHR. The best cut-off value was determined using the Youden index, and was used to defined HcneHR. Uadjusted crude outcomes including NICU mortality, hospital mortality, NICU length of stay and APACHE IV score were compared between HcneHR and LcneHR patients.

Logistic regression model was fitted to adjust to odds ratio of NICU and hospital mortality. Three models were created: model 1 adjusted to APACHE IV score; model 2 adjusted for demographics and APACHE IV score; model 3 adjusted for model 2 covariance plus disease categories, admission vital sign( temperature, respiratory rate, heart rate, mean blood pressure,Sp02 ), the first day hemoglobin, use of mechanical ventilation, use of vasopressors, length of NICU stay, comorbidities, including atrial fibrillation, cardiovascular diseases. A stepwise backward elimination method with a significance level of 0.05 was used to build the final model.

We determined the predictive efficacy of HcneHR in conjunction with currently accepted known risk factors for mortality. Here wo chosen APACHE IV score, use of mechanical ventilation, HcneHR and use of vasopressors to construct prediction model for NICU expired, and showed it in nomogram with R package 
"rms". Each variable was represented by a bar. A given value of a variable can be mapped to the point bar at the top of the graph and there is a point value for that given value. After each variable was assigned to a point number, they were summed and mapped to the total point bar. Then there will be a value in the "Risk of NICU Expired" bar corresponding to those total points. ROC analysis was used to evaluate the predictive ability of the predictive model. For disease subgroup analysis, we used model 1 to calculate odds ratio of NICU and hospital mortality for different diseases, and showed them with forest graph.

Data extraction was performed using PostgreSQL (version 10.5, www.postgresql.org) and pgADmin PostgreSQL tools(version 4). R (version 3.5.1, www.r-project.org) was used for statistical analysis. A twosided $P$ value of $<0.05$ was considered statistically significant.

\section{Results}

\section{Patient Characteristics}

The elCU-CRD contained 200,839 patients, including 14,451 NICU patients. Of the remaining 14,451 patients, we excluded those with length of stay $(24 \mathrm{~h}$, age $<18$, and those who used heart rate controlling drug. A total of 8,347 patients met our inclusion criteria after excluding those with missing data(Fig 1 ), of which 339 were expired when discharged from NICU, giving an NICU mortality rate of $4 \%$. Demographics and baseline characteristics between alive and expired patients are presented in Table 1. The mean age were 63 years, and most patients ethnicity were "Caucasian" (75\%). The main disease categories were postoperative patients $(25 \%)$, stroke $(19 \%)$, traumatic brain injury(TBI) $(14 \%)$, intracranial hemorrhage $(10 \%)$, and so on. The mean APACHE IV score was 50 . On the first $24 \mathrm{hours,}$ cneHR was significantly higher in NICU expired patients than in alive patients( $72.5 \pm 91.58$ vs. $34.26 \pm 65.39 ; p<0.001)$, also in hospital expired and alive patients(71.41 \pm 90.8 vs. $50.52 \pm 73.18 ; p<0.001)$ ( supplementary fig. 1 ). The number of missing data onto hemoglobin was $1127(13.5 \%)$.

\section{HcneHR and the Outcome Analysis}

Table 2 showed crude outcomes by cneHR category. Patients with HcneHR had significantly higher NICU and hospital mortality. ROC analysis showed area under curve(AUC) of the predictive ability of cneHR was 0.655 , and the best cut-off point was 9.5 (supplementary fig.2), that is why we chosen 10 as the cutoff point of HcneHR and LcneHR. The percentage of HcneHR in NICU expired patients was $61 \%$, while the alive one was $36 \%$. Fig. 2 showed the different percentage of HcneHR between NICU alive and expired patients over time. The length of NICU stay and APACHE IV score were significantly different between the HcneHR and LcneHR patients(table 2).

\section{Contribution of HcneHR and other covariance to Mortality}

Table 3 showed the ORs of NICU and hospital mortality in the different logistic regression models. The unadjusted OR of HcneHR for NICU mortality was 2.84(2.27-3.55, $\mathrm{p}<0.001)$. After adjustment for demographics , APACHE IV score and other clinical parameters, the OR decreased but remained 
statistically significant for HcneHR patients. As for subgroup analysis of different diseases, in a univariable logistic regression, HcneHR significantly associated with NICU mortality in postoperation, ischemic stroke, traumatic brain injury, intracranial hemorrhage and others groups. Furthermore, after APACHE IV score was adjusted to a multivariable logistic regression analysis, HcneHR remained significantly associated with NICU mortality in ischemic stroke, intracranial hemorrhage and others groups(fig.3), and significantly associated with hospital mortality in traumatic brain injury, intracranial hemorrhage and others groups (supplementary fig.3).

Fig.4 showed the nomogram of predictive model for prediction of the death risk for NICU patients. The predictive model used APACHE IV score, mechanical ventilation, vasopressors and HcneHR as covariance. ROC analysis of this predictive model showed AUC was 0.855 , as showed in supplementary fig.3.

In a post hoc analysis, we explored the ORs of different heart rate and corresponding cneHR. We calculated the adjusted odds ratio of death using a minimally adjusted logistic regression model (including age, gender, and ethnicity) for each combination of cumulative numbers and heart rate thresholds. The results were shown in Figure 5, where each square showed the corresponding odds ratio of NICU death numerically. There was an increasing trend of OR as the heart rate and cneHR increased.

\section{Discussion}

In this analysis of a large and heterogeneous NICU clinical database, we found a significant association for HcneHR with elevated NICU mortality, hospital mortality, ICU length of stay and APACHE IV score. As cneHR can be readily got from the ECG monitoring system, which is common in NICU, it may be a good tool to better identify high risk patients admitted in NICU. In contrast to other heart rate parameters, cneHR integrates many parameters, like elevated heart rate, volatility and duration.

Elevated resting heart rate (RHR) is associated with all cause mortality in the general population[11, 12], and lower RHR is related to lower all-cause mortality[13]. Elevated preoperative HR is associated with impaired cardiopulmonary performance consistent with clinically unsuspected, subclinical cardiac failure, and is associated with myocardial injury, myocardial infarction, and mortality after non-cardiac surgery $[14,15]$. In critically ill patients, prolonged elevated HR increased incidence of major cardiac events and was associated with a significantly longer ICU stay and reduced survival[5, 16]. Our findings are in line with these studies. That is to say, in critically ill patients admitted in NICU, HcneHR was associated with NICU and hospital mortality. In the time series characteristics of HcneHR percentage, the NICU alive patients had a gradually downward trend, and tended to be stable after 12 hours. However, the HcneHR percentage of NICU expired patients was higher, and jumped over time(Fig.2).

The disease profile of our study contained surgical diagnosis, ischemic stroke, traumatic brain injury,intracranial hemorrhage, seizures, sepsis, subarachnoid hemorrhage, coma, and others groups. There are a number of studies which have evaluated the association for increased HR and mortality in neurocritical patients. Preoperative elevated heart rate is associated with mortality after neurosurgery[15]. 
In moderate and severe TBI patients, a correlation between elevated baseline HR and 60th-day mortality was shown[17]. After SAH, prolonged elevated HR was associated with 3-month poor outcome and an increased hazard for delayed cerebral ischemia, myocardial injury and pulmonary edema[18]. Among intracerebral hemorrhage and ischemic stroke patients, higher admission HR was independently associated with death and poor functional outcome[19, 20]. Elevated HR prior to partial seizure onset of those attacks which become secondarily generalized compared to seizures which remain localized, and this may be relevant to the understanding of sudden death in epilepsy[21]. In our study, subgroup analyses showed HcneHR were associated with NICU mortality in ischemic stroke, intracranial hemorrhage and others groups, and significantly associated with hospital mortality in traumatic brain injury, intracranial hemorrhage and others groups.

There are myriad causes of elevated HR in neurological critically-ill patients, however, including but not limited to pain, agitation, hypovolemia, infection, fever and systemic inflammation, or vasoactive therapy with vasopressors, and multiple potential causes may coexist, making it difficult or impossible to get a precise cause[2-4]. Elevated HR can increase metabolic oxygen demand, increase blood pressure from increased sympathetic activity, activate inflammatory pathway, or cause endothelial dysfunction[22, 23]. The precise mechanism between elevated HR and poor outcome still remain difficult to unravel.

This was an observational analysis and therefore we cannot draw causal conclusions, and other unknown confounders may influence the result. It is of paramount importance to analyze covariance in a retrospective cohort study. We attempted to include a wide range of clinically important confounders to reach valid results and performed PSM to account for group differences. We used the multivariable logistic regression to adjust several clinically important confounders, like age, gender, ethnicity, temperature, mean blood pressure, $\mathrm{SpO} 2$, mechanical ventilation use, vasopressors use, comorbid heart disease, admission hemoglobin and so on. This association was maintained after adjusting to these clinically important confounders. APACHE IV scoring system had good discriminatory capability for critical patients[24, 25]. In the multivariable logistic regression analysis, we found that HcneHR was associated with NICU and hospital mortality after APACHE IV score was adjusted, indicating that HcneHR may provide prognostic information independent of APACHE IV score. The increasing trend of OR as the heart rate and cneHR increased may suggested dose-response relationship.The finding can help with prognosis prediction and raise the question about a causal relationship.

Our findings posed the question as to whether therapeutic reduction of HR is beneficial to neurocritical patients. For patients in septic shock, $\beta$-blocker was associated with reductions in heart rates to achieve target levels, without increased adverse events, and with a lower twenty-eight day mortality[26]. A metaanalysis of nine observational studies showed in adults with acute TBI, exposure to $\beta$-blockers after TBI was associated with a reduction of in-hospital mortality (pooled OR $0.39,95 \% \mathrm{Cl}: 0.27-0.56 ; 12=65 \%$, $p<0.00001)[27]$. There is a growing body of evidence showing that perioperative $\beta$-blocker exposure was associated with lower rates of 30-day all-cause mortality in cardiac high-risk patients undergoing noncardiac surgery[28]. Nevertheless, the right timeframe for intervention and the appropriate heart rate 
goals are currently undefined. Further studies are required to investigate strategies for HR control in NICU patients. Appropriately randomized controlled multi center trials are required to confirm these questions.

The neurocritical care unit is a dataintensive environment. Utilization of these dense data onto realtime decisionmaking for patient care represents the art of neurocritical care practice[7]. Despite advances in medicine, data onto vital signs of bedside monitors in neurocritical care are evaluated by clinicians almost in much the same way as before. In one study of NICU patients, the investigator rather than just displayed the maximum daily temperature as the measure of fever, calculated the area under the curve (AUC) above a specific cutoff (such as $>38.5^{\circ} \mathrm{C}$ ) and provided a morerobust measure of 'dose' of fever[29]. In Olaf Sander's study prolonged elevated HR (PEHR) group was defined as $>95 \mathrm{beats} / \mathrm{min}$ for $>12$ hours in at least one 24-hour period of ICU stay starting at admission[5]. And in Veit Sandfort's study a prolonged eHR (peHR) episode was prespecified as 11 hourly heart rate measurements $>100$ beats $/$ min in any given 12-hour interval [16]. These are examples of 'dose' parameters. In other studies, measures of $\mathrm{HR}$ volatility (standard deviation, \% HR $>120 \mathrm{bpm}$, and \%HR<60 bpm) were used to predict prognosis[30, 31]. In our study, cneHR was used as an index to evaluate HR, it was also a 'dose' and 'volatility' parameter. It can reflex duration of elevated HR, on the flip side may present HR volatility. Most importantly, cneHR can be displayed in real time at the bedside because it can be acquired and calculated automatically.

In general, the large sample size, inclusion of multiple NICUs, and the excellent quality of the clinical data collection by the treatment centers, for example, the periodic heart rate is available for $96 \%$ of patients, are advantages of this analysis. The chosen variable to define 'dose' and 'volatility'of elevated HR was found to be suitable to identify patients with an increased risk of mortality in the NICU. However, there are several limitations of our study. Firstly, the study is retrospective, its post hoc nature should be taken into account when considering the findings. Residual confounding may also influence our findings, although we attempted to account for this through several adjustments and different models. Secondly, although the datasets we used are large and comprise routinely collected clinical data, some patients had to be excluded owing to poor-quality data recording or missing data. The amount of missing data onto the hemoglobin assessed in the study is a potential limitation. In the stepwise regression analysis, hemoglobin was contained in the final model. Because of the missing data, we dropped it in the predictive model. Thirdly, in elCU-CRD, all patients admitted from the operating room or recovery room had a surgical diagnosis, even though some surgical patients have a medical reason for admission. That may to some extent change disease spectrum. Although our findings do support an association with HcneHR and NICU mortality, stronger evidence is necessary to establish a causal relationship.

\section{Conclusions}

In adult neurocritically ill patients, HcneHR in the first day of NICU stay is independently associated with higher NICU mortality and several others important patient-centered outcomes.

\section{Abbreviations}


NICUヌNeurological intensive care unit

HcneHR: high cumulative numbers of elevated Heart Rate

elCU-CRD: elCU Collaborative Research Database

APACHE: Acute Physiology and Chronic Health Evaluation

IRB: institutional review board Sp02: pulse oxygen saturation

NOCB: observation carried backward ROC: receiver operating characteristic

TBI: traumatic brain injury AUC: area under curve

\section{Declarations}

\section{Acknowledgments}

We thank the anonymous reviewers for insightful and helpful comments.

Funding:This study was supported by grants from key support projects of "Yangfan Plan" of Beijing Medical Administration(NO.ZYLX201836).

\section{Availability of data and materials}

The datasets generated and analysed during the current study are available in the elCU Collaborative Research Database(eicu-crd.mit.edu).

\section{Authors' contributions}

DWZ, JXZ and GZS were responsible for the concept and participated the design of the study. DWZ, ZML and SLZ participated the data collection and the data analysis and interpretation. DWZ, JXZ and GZS participated the statistical analysis. DWZ and GZS wrote the first draft of the manuscript. All authors commented on the manuscript revisions and approved the final version.

\section{Ethics approval and consent to participate}

The eICU-CRD was exempt from institutional review board approval due to the retrospective design, lack of direct patient intervention, and the security schema, for which the re-identification risk was certified as meeting safe harbor standards by Privacert (Cambridge, MA) (Health Insurance Portability and Accountability Act Certification no. 1031219-2). Due to the HIPAA compliant de-identification in this database, our institutional IRB requirement was waived. Patient's consents were waived due to the retrospective design of the study.

Consent for publication: Not applicable. 


\section{Competing interests}

The authors declare that they have no competing interests.

\section{References}

1. Adhikari, N.K.J., Fowler, R.A., Bhagwanjee, S.Rubenfeld, G.D. Critical care and the global burden of critical illness in adults. The Lancet 2010;376(9749):1339-46.

2. Verrier, R.L.Tan, A. Heart rate, autonomic markers, and cardiac mortality. Heart Rhythm 2009;6(11 Suppl):S68-75.

3. Fox, K.M.Ferrari, R. Heart rate: a forgotten link in coronary artery disease? Nat Rev Cardiol 2011;8(7):369-79.

4. Caetano, J.Delgado Alves, J. Heart rate and cardiovascular protection. Eur J Intern Med 2015;26(4):217-22.

5. Sander, O., Welters, I.D., Foëx, P.Sear, J.W. Impact of prolonged elevated heart rate on incidence of major cardiac events in critically ill patients with a high risk of cardiac complications*. Critical Care Medicine 2005;33(1):81-8.

6. Zimmerman, J.E., Kramer, A.A., McNair, D.S.Malila, F.M. Acute Physiology and Chronic Health Evaluation (APACHE) IV: hospital mortality assessment for today's critically ill patients. Crit Care Med 2006;34(5):1297-310.

7. Hemphill, J.C., Andrews, P.De Georgia, M. Multimodal monitoring and neurocritical care bioinformatics. Nat Rev Neurol 2011;7(8):451-60.

8. Moheet, A.M., Livesay, S.L., Abdelhak, T., et al. Standards for Neurologic Critical Care Units: A Statement for Healthcare Professionals from The Neurocritical Care Society. Neurocritical Care 2018;29(2):145-60.

9. Pollard, T.J., Johnson, A.E.W., Raffa, J.D., et al. The elCU Collaborative Research Database, a freely available multi-center database for critical care research. Sci Data 2018;5:180178.

10. Nelson, L.S. The Anderson-Darling test for normality. Journal of Quality Technology 1998;30(3):298-9.

11. Aladin, A.l., Whelton, S.P., Al-Mallah, M.H., et al. Relation of resting heart rate to risk for all-cause mortality by gender after considering exercise capacity (the Henry Ford exercise testing project). Am J Cardiol 2014;114(11):1701-6.

12. Sharashova, E., Wilsgaard, T., Løchen, M.-L., et al. Resting heart rate trajectories and myocardial infarction, atrial fibrillation, ischaemic stroke and death in the general population: The Troms $\varnothing$ Study. European Journal of Preventive Cardiology 2017;24(7):748-59. 
13. Saxena, A., Minton, D., Lee, D.C., et al. Protective role of resting heart rate on all-cause and cardiovascular disease mortality. Mayo Clin Proc 2013;88(12):1420-6.

14. Abbott, T.E.F., Minto, G., Lee, A.M., et al. Elevated preoperative heart rate is associated with cardiopulmonary and autonomic impairment in high-risk surgical patients. Br J Anaesth 2017;119(1):8794.

15. Abbott, T.E., Ackland, G.L., Archbold, R.A., et al. Preoperative heart rate and myocardial injury after noncardiac surgery: results of a predefined secondary analysis of the VISION study. Br J Anaesth $2016 ; 117(2): 172-81$.

16. Sandfort, V., Johnson, A.E.W., Kunz, L.M., Vargas, J.D.Rosing, D.R. Prolonged Elevated Heart Rate and 90-Day Survival in Acutely III Patients: Data From the MIMIC-III Database. J Intensive Care Med 2018:885066618756828.

17. Mahsa Hasanpour Mir, F.Y., Mohammad Abdollahi, Arezoo Ahmadi, Atabak Nadjafi The predictive value of resting heart rate following osmotherapy in brain injury: back to basics. DARU Journal of Pharmaceutical Sciences 2012;20:102.

18. Schmidt, J.M., Crimmins, M., Lantigua, H., et al. Prolonged elevated heart rate is a risk factor for adverse cardiac events and poor outcome after subarachnoid hemorrhage. Neurocrit Care 2014;20(3):390-8.

19. Qiu, M., Sato, S., Zheng, D., et al. Admission Heart Rate Predicts Poor Outcomes in Acute Intracerebral Hemorrhage: The Intensive Blood Pressure Reduction in Acute Cerebral Hemorrhage Trial Studies. Stroke 2016;47(6):1479-85.

20. Erdur, H., Scheitz, J.F., Grittner, U., et al. Heart rate on admission independently predicts in-hospital mortality in acute ischemic stroke patients. Int J Cardiol 2014;176(1):206-10.

21. Nilsen, K.B., Haram, M., Tangedal, S., Sand, T.Brodtkorb, E. Is elevated pre-ictal heart rate associated with secondary generalization in partial epilepsy? Seizure 2010;19(5):291-5.

22. Whelton, S.P., Narla, V., Blaha, M.J., et al. Association between resting heart rate and inflammatory biomarkers (high-sensitivity C-reactive protein, interleukin-6, and fibrinogen) (from the Multi-Ethnic Study of Atherosclerosis). Am J Cardiol 2014;113(4):644-9.

23. Whelton, S.P., Blankstein, R., Al-Mallah, M.H., et al. Association of resting heart rate with carotid and aortic arterial stiffness: multi-ethnic study of atherosclerosis. Hypertension 2013;62(3):477-84.

24. Moreno, V.a. Clinical review: Scoring systems in the critically ill. Critical Care Medicine 2010;14:207.

25. Keegan, M.T., Gajic, O.Afessa, B.Comparison of APACHE III, APACHE IV, SAPS 3, and MPMOIII and influence of resuscitation status on model performance. Chest 2012;142(4):851-8. 
26. Morelli, A., Ertmer, C., Westphal, M., et al. Effect of heart rate control with esmolol on hemodynamic and clinical outcomes in patients with septic shock: a randomized clinical trial. JAMA 2013;310(16):1683-91.

27. Alali, A.S., Mukherjee, K., McCredie, V.A., et al. Beta-blockers and Traumatic Brain Injury: A Systematic Review, Meta-analysis, and Eastern Association for the Surgery of Trauma Guideline. Ann Surg 2017;266(6):952-61.

28. London MJ, H.K., Schwartz GG, Henderson WG. Association of perioperative $\beta$-blockade with mortality and cardiovascular morbidity following major noncardiac surgery. JAMA 2013;309(16):1704-13.

29. Diringer, M.N.Neurocritical Care Fever Reduction Trial, G. Treatment of fever in the neurologic intensive care unit with a catheter-based heat exchange system. Crit Care Med 2004;32(2):559-64.

30. Grogan, E.L., Norris, P.R., Speroff, T., et al. Volatility: A New Vital Sign Identified Using a Novel Bedside Monitoring Strategy. The Journal of Trauma: Injury, Infection, and Critical Care 2005;58(1):7-14.

31. Norris, P.R., Ozdas, A., Cao, H., et al. Cardiac uncoupling and heart rate variability stratify ICU patients by mortality: a study of 2088 trauma patients. Ann Surg 2006;243(6):804-12; discussion 12-4.

\section{Strobe Statement}

STROBE Statement-checklist of items that should be included in reports of observational studies 
Item Recommendation

No.
Page No.

Relevant text from manuscript

Title and abstract

1

(a) Indicate the study's design with a commonly used term in the title or the abstract

(b) Provide in the abstract an informative and balanced summary of what was done and what was found

Page-3 and Page-

4

Page-1 Title

Introduction
Background/rationale 2 Explain the scientific background and Page-4 Introduction rationale for the investigation being reported

Objectives

3

State specific objectives, including any prespecified hypotheses

Page-5 Introduction

Methods

Study design

$4 \quad$ Present key elements of study design early in the paper

Page-5, 6, Methods

7,8 and

Page-9

Setting

5 Describe the setting, locations, and relevant dates, including periods of recruitment, exposure, follow-up, and data collection

Participants

6

(a) Cohort study-Give the eligibility criteria, and the sources and methods of selection of participants. Describe methods of follow-up

Page-5

Methods:

Abstract

Case-control study-Give the eligibility criteria, and the sources and methods of case ascertainment and control selection. Give the rationale for the choice of cases and controls

Cross-sectional study-Give the eligibility criteria, and the sources and methods of selection of participants

$\begin{array}{ll}\text { Page-6 } & \text { Methods: } \\ & \text { Study } \\ & \text { Population }\end{array}$

(b) Cohort study-For matched studies, give matching criteria and number of exposed and unexposed

Not applicable

Case-control study-For matched studies, give matching criteria and the number of controls per case

Variables

$7 \quad$ Clearly define all outcomes, exposures,

Page-6

Methods predictors, potential confounders, and 
effect modifiers. Give diagnostic criteria, if applicable

$\begin{array}{llll}\text { Data sources/ } & 8^{*} & \begin{array}{l}\text { For each variable of interest, give } \\ \text { measurement } \\ \text { of assessment (measurement). } \\ \text { Describe comparability of assessment } \\ \text { methods if there is more than one } \\ \text { group }\end{array} & \text { Page-7 }\end{array}$

$\begin{array}{llll}9 & \begin{array}{l}\text { Describe any efforts to address } \\ \text { potential sources of bias }\end{array} & \begin{array}{l}\text { Page-15 } \\ \text { and Page- } \\ 16\end{array} & \text { Discussion }\end{array}$

$\begin{array}{llll}\text { Study size } & 10 \quad \text { Explain how the study size was arrived Page-6 }\end{array}$
at

Quantitative variables $11 \quad \begin{aligned} & \text { Explain how quantitative variables were } \\ & \text { handled in the analyses. If applicable, }\end{aligned}$ describe which groupings were chosen and why

\begin{tabular}{lcll} 
Statistical methods 12 & $\begin{array}{l}\text { (a) Describe all statistical methods, } \\
\text { including those used to control for } \\
\text { confounding }\end{array}$ & $\begin{array}{l}\text { Page-7,8 } \\
\text { and Page- } \\
9\end{array}$ & $\begin{array}{l}\text { Statistical } \\
\text { Analysis }\end{array}$ \\
$\begin{array}{lll}\text { (b) Describe any methods used to } \\
\text { examine subgroups and interactions }\end{array}$ & $\begin{array}{l}\text { Page-8 } \\
\text { and Page- } \\
9\end{array}$ & $\begin{array}{l}\text { Statistical } \\
\text { Analysis }\end{array}$ \\
& $\begin{array}{l}\text { Page-8 } \text { Explain how missing data were } \\
\text { addressed }\end{array}$ & $\begin{array}{l}\text { Statistical } \\
\text { Analysis }\end{array}$ \\
$\begin{array}{l}\text { (d) Cohort study-If applicable, explain } \\
\text { how loss to follow-up was addressed } \\
\text { Case-control study-If applicable, } \\
\text { explain how matching of cases and } \\
\text { controls was addressed }\end{array}$ & $\begin{array}{l}\text { Not } \\
\text { applicable } \\
\text { Cross-sectional study-If applicable, } \\
\text { describe analytical methods taking } \\
\text { account of sampling strategy }\end{array}$ & \\
\hline
\end{tabular}
(e) Describe any sensitivity analyses
$\mathrm{N} / \mathrm{a}$

Results

Participants

$13^{*}$

(a) Report numbers of individuals at each stage of study-eg numbers potentially eligible, examined for eligibility, confirmed eligible, included in the study, completing follow-up, and analysed

$\begin{array}{ll}\text { Page-7 } & \text { Statistical } \\ \text { Analysis }\end{array}$

$\begin{array}{ll}\text { Page-7,8 } & \text { Statistical } \\ \text { and Page- } & \text { Analysis } \\ 9 & \end{array}$

$\begin{array}{ll}\text { Page-8 } & \text { Statistical } \\ \text { and Page- } & \text { Analysis } \\ 9 & \end{array}$

Not

(d) Cohort study-If applicable, explain

(

(b) Give reasons for non-participation at each stage

Figure-1

(c) Consider use of a flow diagram

Figure-1 
(a) Give characteristics of study

Page-9

Results participants (eg demographic, clinical, social) and information on exposures and potential confounders

(b) Indicate number of participants with Page-10 Results missing data for each variable of interest

(c) Cohort study-Summarise follow-up N/a time (eg, average and total amount)

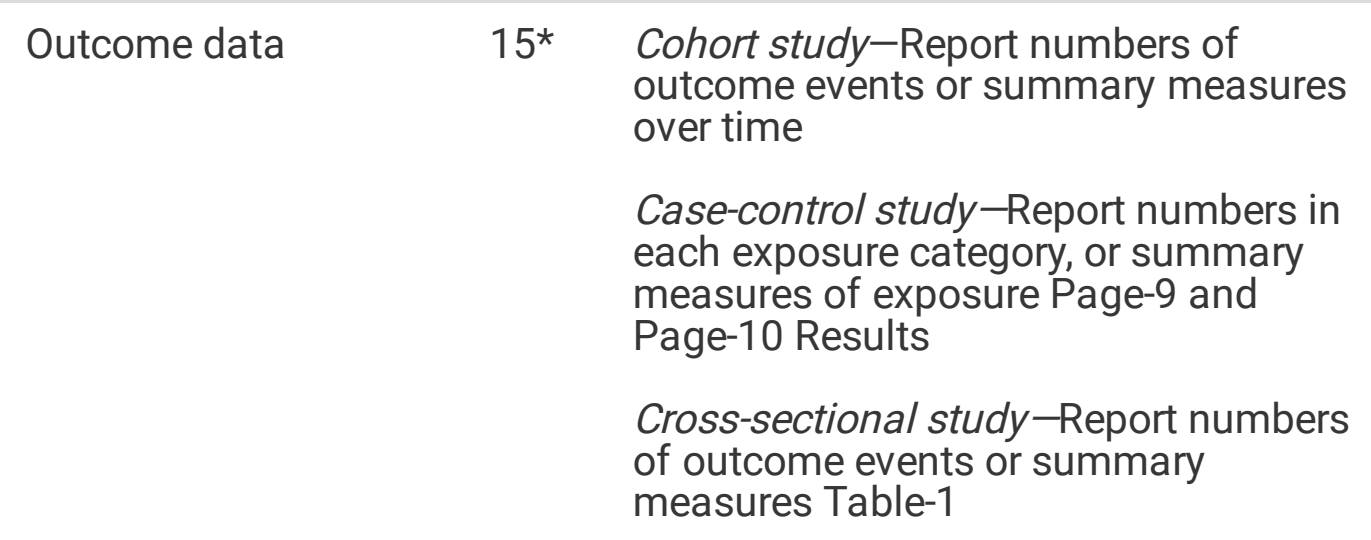

Main results

16

(a) Give unadjusted estimates and, if applicable, confounder-adjusted

Page-10, Results estimates and their precision (eg, 95\% confidence interval). Make clear which confounders were adjusted for and why they were included

(b) Report category boundaries when continuous variables were categorized

Page-7

Methods

(c) If relevant, consider translating $\mathrm{N} / \mathrm{a}$ estimates of relative risk into absolute risk for a meaningful time period Table-2

Continued on next page 
Other analyses

17 Report other analyses done-eg analyses of subgroups and interactions, and sensitivity analyses
Page-11, Results Figure-3

\section{Discussion}

Key results

18 Summarise key results with reference to study objectives

Page-11 Discussion

and

Page-12

Limitations

19 Discuss limitations of the study, taking into account sources of potential bias or imprecision. Discuss both direction and magnitude of any potential bias

Page-15 Discussion

and

Page-16

Interpretation

20 Give a cautious overall interpretation of results considering objectives, limitations, multiplicity of analyses, results from similar studies, and other relevant evidence

Page-12,

Discussion

13,14 ,

15 and

Page-16

Generalisability

21

Discuss the generalisability (external validity) of the study results

Page-15 and

Page-16

\section{Other}

information

Funding

22 Give the source of funding and the role of the funders for the present study and, if applicable, Page-16 Funding for the original study on which the present article is based

*Give information separately for cases and controls in case-control studies and, if applicable, for exposed and unexposed groups in cohort and cross-sectional studies.

Note: An Explanation and Elaboration article discusses each checklist item and gives methodological background and published examples of transparent reporting. The STROBE checklist is best used in conjunction with this article (freely available on the Web sites of PLoS Medicine at http://www.plosmedicine.org/, Annals of Internal Medicine at http://www.annals.org/, and Epidemiology at http://www.epidem.com/). Information on the STROBE Initiative is available at www.strobestatement.org.

\section{Tables}

Table 1 Comparison of baseline characteristics between alive and expired patients 
Variables

Total $(\mathrm{n}=8347) \quad$ Alive $(\mathrm{n}=8008)$

Expired $(n=339)$

P

value

Age,years (meadian,[IQR])

63(51,75)

63(51,75)

$67(54,77)$

0.002

Sex:male(n(\%))

4312(52)

4118(51)

194(57)

0.041

Admission vital sign

Temperature,C(meanSD)

$36.47 \pm 0.82$

$36.47 \pm 0.79$

$36.37 \pm 1.42$

0.18

Respiratory rate(meanSD)

$23.21 \pm 14.45$

$23.1 \pm 14.43$

$25.98 \pm 14.49$

$<0.001$

HR,beats/min(meanSD)

$\mathrm{MBP}, \mathrm{mmHg}$ (meanSD)

$97.05 \pm 30.45$

$96.4 \pm 30.22$

$112.3 \pm 31.88$

$<0.001$

Sp02,\%(meadian,[IQR])

$99.17 \pm 42.67$

$99.31 \pm 42.45$

$96.01 \pm 47.61$

0.211

$98(96,100)$

$98(96,100)$

$99(96,100)$

$<0.001$

Heart Rate related indexes for continuously monitoring

\begin{tabular}{lllll}
\hline cneHR (meadian,[IQR]) & $35.81 \pm 67.07$ & $34.26 \pm 65.39$ & $72.5 \pm 91.58$ & $<0.001$ \\
\hline HcneHR (n(\%)) & $3082(37)$ & $2874(36)$ & $208(61)$ & $<0.001$ \\
\hline MaxHR,beats/min(meanSD) & $105.17 \pm 20.25$ & $104.65 \pm 19.91$ & $117.48 \pm 23.96$ & $<0.001$ \\
\hline MinHR,beats/min(meanSD) & $65.19 \pm 14.94$ & $65.01 \pm 14.83$ & $69.56 \pm 16.88$ & $<0.001$ \\
\hline AHR,beats/min(meanSD) & $80.92 \pm 15.11$ & $80.57 \pm 14.93$ & $89.23 \pm 16.92$ & $<0.001$ \\
\hline APS (meanSD) & $38.59 \pm 20.96$ & $37.26 \pm 19.42$ & $70.09 \pm 29.6$ & $<0.001$ \\
\hline APACHE IV score(meanSD) & $49.61 \pm 22.82$ & $48.21 \pm 21.44$ & $82.53 \pm 28.87$ & $<0.001$ \\
\hline Hemoglobin,g/dl(meanSD) & $11.79 \pm 2.02$ & $11.8 \pm 2$ & $11.57 \pm 2.38$ & 0.077 \\
\hline Ethnicity(n(\%)) & & & & 0.355 \\
\hline Caucasian & $6298(75)$ & $6053(76)$ & $245(72)$ & \\
\hline African American & $1128(14)$ & $1077(13)$ & $51(15)$ & \\
\hline Hispanic & $287(3)$ & $278(3)$ & $9(3)$ & \\
\hline Asian & $128(2)$ & $122(2)$ & $6(2)$ & \\
\hline ative American & $59(1)$ & $57(1)$ & $2(1)$ & \\
\hline Other/Unknown & $447(5)$ & $421(5)$ & $26(8)$ & \\
\hline Disease category(n(\%)) & & & $51(15)$ & \\
\hline Postoperation & $2111(25)$ & $2060(26)$ & $1516(19)$ & 601 \\
\hline Ischemic stroke & $1576(19)$ & & & \\
\hline
\end{tabular}




\begin{tabular}{lllll} 
Traumatic brain injury & $1197(14)$ & $1150(14)$ & $47(14)$ & \\
\hline Intracranial hemorrhage & $799(10)$ & $731(9)$ & $68(20)$ & \\
\hline Seizures & $373(4)$ & $365(5)$ & $8(2)$ & \\
\hline Sepsis & $283(3)$ & $255(3)$ & $28(8)$ & \\
\hline Subarachnoid hemorrhage & $233(3)$ & $218(3)$ & $15(4)$ & \\
\hline Coma & $179(2)$ & $161(2)$ & $18(5)$ & 0.078 \\
\hline Others & $1596(19)$ & $1552(19)$ & $44(13)$ & 0.001 \\
\hline Atrial fibrillation(n(\%)) & $229(3)$ & $214(3)$ & $15(4)$ & $<0.001$ \\
\hline Cardiovascular Diseases(n(\%)) & $2395(29)$ & $2269(28)$ & $126(37)$ & $<0.001$ \\
\hline Tracheal intubation(n(\%)) & $1290(15)$ & $1123(14)$ & $167(49)$ & $<0.001$ \\
\hline Mechanical ventilation(n(\%)) & $1880(23)$ & $1655(21)$ & $225(66)$ & $<0.001$
\end{tabular}

IQR interquatile range, SD standard deviation,HR heart rate,HcneHR high cumulative numbers of elevated Heart Rate,MaxHR maximum heart rate, MinHR minimum heart rate, AHR average heart rate,Sp02 Pulse Oxygen Saturation,APS Acute physiology score, APACHE Acute Physiology and Chronic Health Evaluation, NICU neurologic intensive care unit, LOS length of stay

Table 2 Crude primary and other outcomes between HcneHR and LcneHR patients

\begin{tabular}{lllll} 
Variables & Total $(\mathrm{n}=8347)$ & LcneHR(n=5265) & HcneHR(n=3082) & P value \\
\hline NICU Expired(n(\%)) & $339(4)$ & $131(2)$ & $208(7)$ & $<0.001$ \\
\hline Hospital Expired(n(\%)) & $669(8)$ & $295(6)$ & $374(12)$ & $<0.001$ \\
\hline NICU LOS(days) (meanSD) & $4.17 \pm 4.75$ & $3.83 \pm 4.3$ & $4.76 \pm 5.39$ & $<0.001$ \\
\hline APS(meanSD) & $38.59 \pm 20.96$ & $34.08 \pm 18.06$ & $46.29 \pm 23.2$ & $<0.001$ \\
\hline APACHE IV score(meanSD) & $49.61 \pm 22.82$ & $45.58 \pm 20.5$ & $56.48 \pm 24.86$ & $<0.001$ \\
\hline Tracheal intubation(n(\%)) & $1290(15)$ & $605(11)$ & $685(22)$ & $<0.001$ \\
\hline Mechanical ventilation(n(\%)) & $1880(23)$ & $943(18)$ & $937(30)$ & $<0.001$ \\
\hline Vasopressors $(\mathrm{n}(\%))$ & $371(4)$ & $164(3)$ & $207(7)$ & $<0.001$
\end{tabular}

HcneHR high cumulative numbers of elevated Heart Rate, LcneHR low cumulative numbers of elevated Heart Rate, NICU neurologic intensive care unit, LOS length of stay, APS Acute physiology score, APACHE 
Acute Physiology and Chronic Health Evaluation.

Table 3 Unadjusted and adjusted odds ratio for NICU and hospital mortality by cneHR
Model
HcneHR for NICU
HcneHR for
mortality
hospital mortality
OR $(95 \% \mathrm{Cl})$
P value
OR $(95 \% \mathrm{CI})$
P value

Unadjusted $\quad 2.84(2.27-3.55)$
$<0.001$
$2.33(1.98-2.73)$
$<0.001$

Model 1

$1.62(1.27-2.06)$

$$
<0.001
$$

$1.42(1.19-1.69)$

$<0.001$

Model 2

1.59 (1.27-2.04)

$$
<0.001
$$

$<0.001$

Model 3

0.014

$1.50(1.09-2.08)$
$1.37(1.09-1.74)$

0.008

$<0.001$

$<0.001$

Predictive

model
1.39(1.16-1.65)
$<0.001$

$<0.001$
$1.50(1.24-1.79)$

$1.57(1.23-2.01)$

$(2)<0.001$ 
Supplementary fig. 2: ROC analysis of cneHR for NICU mortality

Supplementary fig. 3: Odds Ratio of different disease subgroups for hospital expired patients

Supplementary fig. 4: ROC analysis of predictive model

\section{Figures}

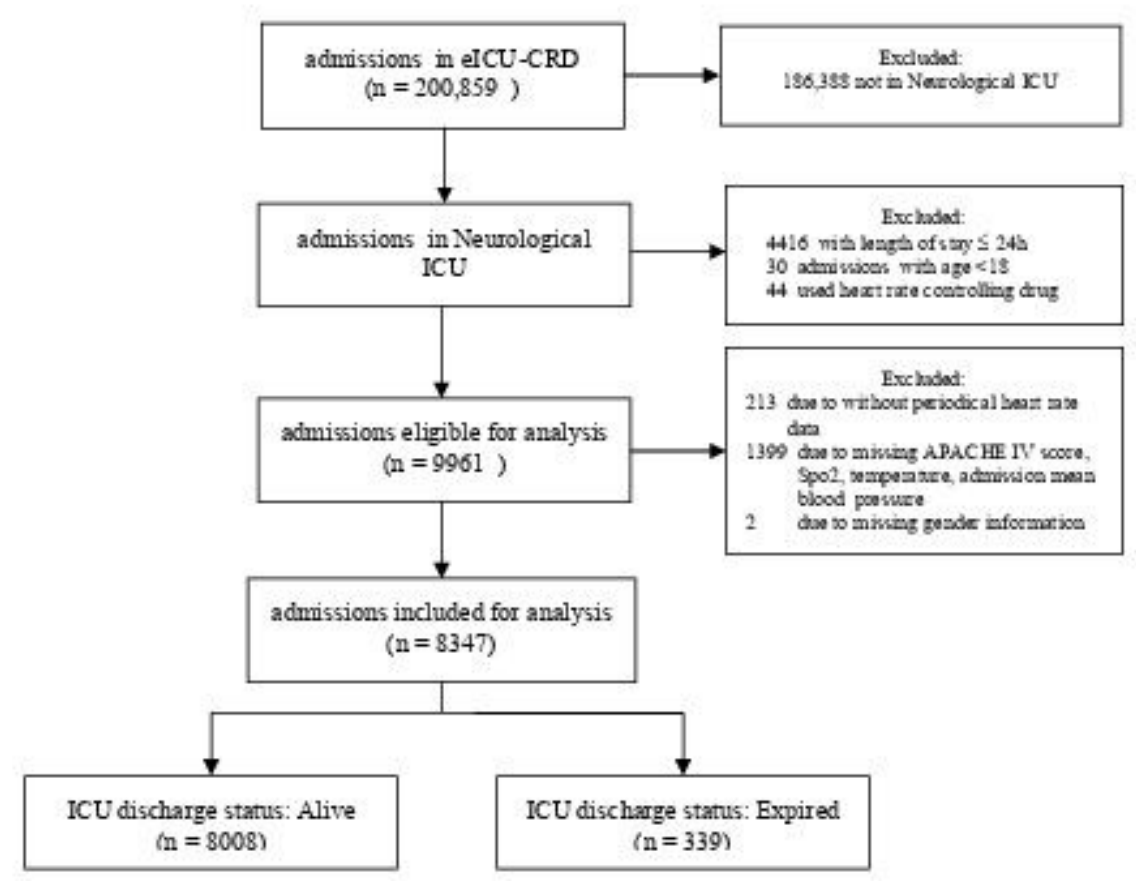

Figure 1

Flow chart of subject selection 


\section{Different Percentage of HcneHR between NICU Alive and Expired patients}

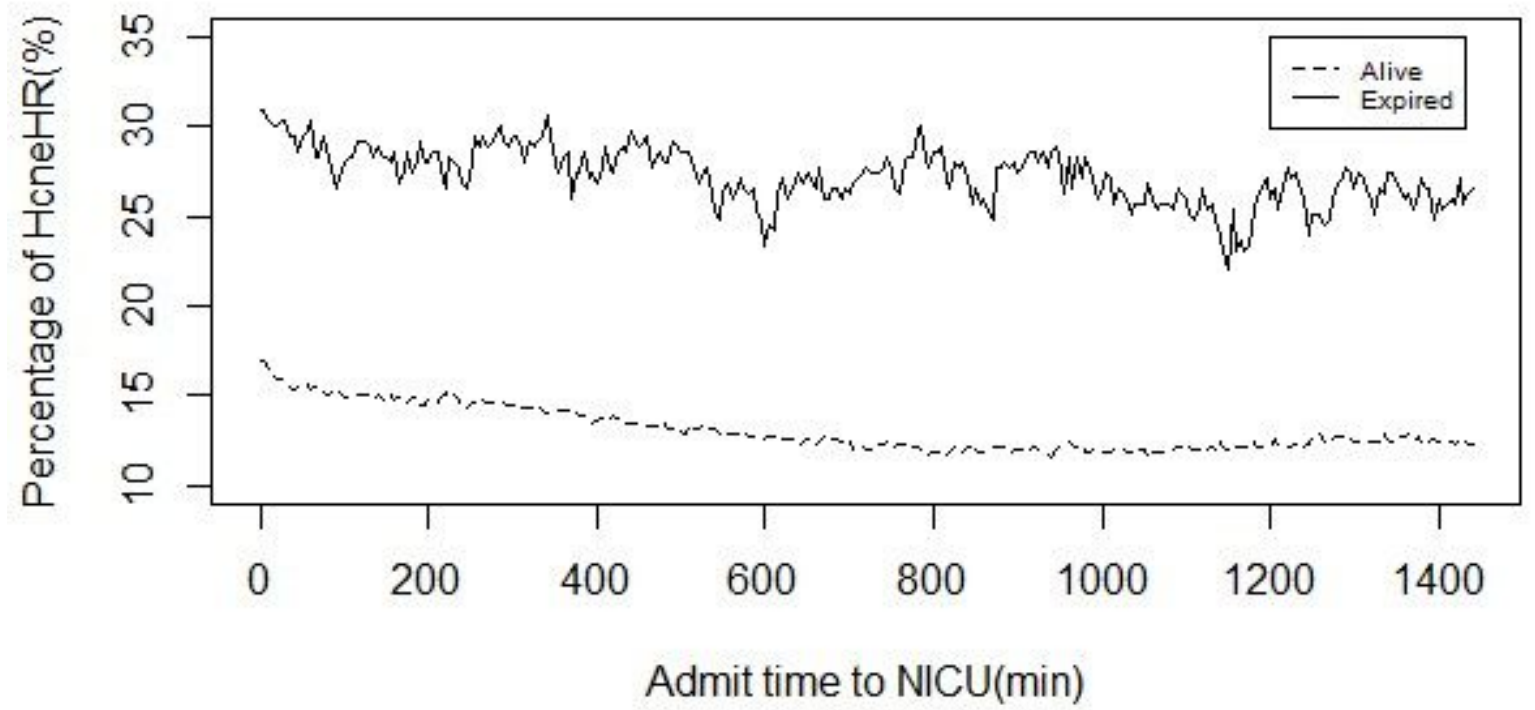

Figure 2

different percentage of HcneHR between NICU alive and expired patients over time

\begin{tabular}{|c|c|c|c|}
\hline Diagnoses & & $\mathrm{OR}(95 \% \mathrm{Cl})$ & $P$ value \\
\hline Postoperation & - & $1.64(0.89-3.06)$ & 0.112 \\
\hline Ischemic stroke & $=$ & $1.86(1.06-3.3)$ & 0.031 \\
\hline Traumatic brain injury & 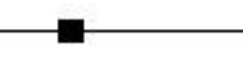 & $1.74(0.86-3.58)$ & 0.125 \\
\hline Intracranial hemorrhage & - & $1.72(0.99-3)$ & 0.054 \\
\hline Seizures & & $0.97(0.21-4.37)$ & 0.965 \\
\hline Sepsis & & $1.23(0.47-3.69)$ & 0.685 \\
\hline Subarachnoid hemorrhage & & $1.16(0.33-3.92)$ & 0.814 \\
\hline Coma & & $1.02(0.35-2.89)$ & 0.971 \\
\hline Others & $E$ & $1.94(1.03-3.77)$ & 0.042 \\
\hline Total & $\rightarrow-$ & $1.62(1.27-2.06)$ & $<0.001$ \\
\hline
\end{tabular}


Figure 3

Odds Ratio of different disease subgroups for NICU expired patients

Points

APACHEIV

MV

HcneHR

Vasopressors

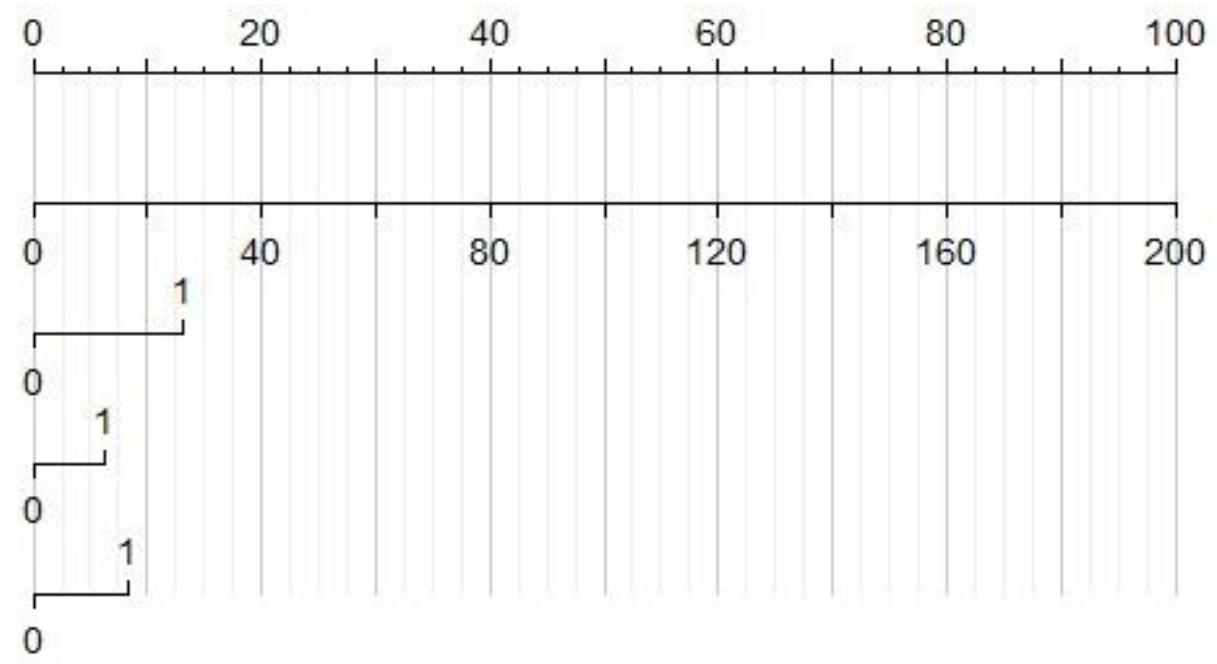

Total Points

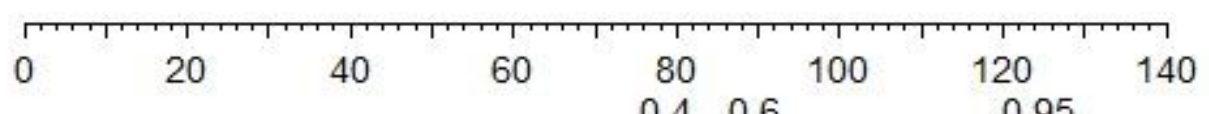

Risk of NICU Expired

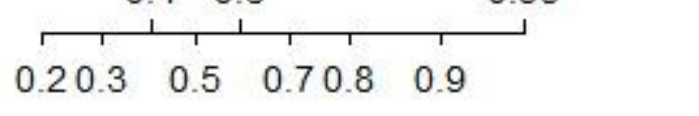

\section{Figure 4}

Nomogram of predictive model for the risk of death for NICU patients 


\section{ORs for various heart rate and cumulative numbers}

\begin{tabular}{|c|c|c|c|c|c|c|}
\hline $125^{-}$ & 3.87 & 4. 42 & 4. 33 & 4. 55 & 4. 88 & 4. 87 \\
\hline $120-$ & 3.48 & 3. 77 & 3.65 & 4. 04 & 4. 02 & 4. 09 \\
\hline $115^{-}$ & 3.2 & 3.31 & 3. 64 & 3.38 & 3. 61 & 3. 85 \\
\hline $110^{-}$ & 3.29 & 3.35 & 3.3 & 3.26 & 3.48 & 3. 41 \\
\hline $105^{-}$ & 2. 88 & 3. 11 & 3. 24 & 3.06 & 2. 97 & 3.08 \\
\hline $100-$ & 2.95 & 2.7 & 2. 74 & 2. 76 & 2. 89 & 2.8 \\
\hline & 1 & | & | & | & 1 & 1 \\
\hline & 10 & 20 & 30 & 40 & 50 & 60 \\
\hline & Cumula & e Num & $S$ fol & rres & ing $\mathrm{H}$ & Rate \\
\hline
\end{tabular}

Figure 5

odds ratio for various heart rate and cumulative numbers over corresponding heart rate

\section{Supplementary Files}

This is a list of supplementary files associated with this preprint. Click to download.

- Supplementaryfig.1.tiff

- Supplementaryfig.4.tiff 
- Supplementaryfig.3.tiff

- Supplementaryfig.2.tiff 